\title{
Relationship of regression pattern to recurrence in retinoblastoma
}

\author{
A D Singh, D Garway-Heath, S Love, P N Plowman, J E Kingston, J L Hungerford
}

\begin{abstract}
A retrospective analysis has been conducted of regression patterns following treatment of retinoblastoma by external beam irradiation. There were 180 tumours in 105 eyes of 83 patients. Type I regression was found to be the commonest pattern and occurred in $50 \%$ of cases. Initial tumour size was found to be the only statistically significant determinant of regression pattern $(\mathbf{p}<0 \cdot 01)$. Thirteen tumours (7\%) recurred within a median interval to recurrence of 12 months. All recurrences occurred within 40 months of completion of treatment and none occurred after age 4 years. No tumour less than $6 \mathrm{~mm}$ in diameter recurred. Although 10 out of 13 recurrences were of Type I, Cox model regression analysis showed initial tumour size to be the only independent predictor of recurrence $(\mathbf{p}<0.01)$. (BrF Ophthalmol 1993; 77: 12-16)
\end{abstract}

The past 30 years has seen a trend towards conservative treatment of retinoblastoma. ${ }^{12}$ Retinoblastoma is a highly radiosensitive tumour $^{2-4}$ and external beam irradiation (EBI) has been used successfully since $1903 .{ }^{5}$ Although the majority of tumours regress after EBI, approximately $25 \%$ of patients require additional therapy because a tumour fails to regress or recurs, or because one or more new tumours arise.

Retinoblastoma tumours which respond to

Department of Ophthalmology A D Singh

D Garway-Heath

$\mathrm{J} L \mathrm{~L}$ Hungerford

Department of

Radiotherapy

P N Plowman

Department of Paediatric Oncology, St

Bartholomew's Hospital, West Smithfield, London J E Kingston

Moorfields Eye Hospital, City Road, London

$\mathrm{J} L \mathrm{~L}$ Hungerford

Department of Medical Statistics, Imperial Cancer Research Fund, Lincoln's Inn Fields, London

S Love

Correspondence to:

J L Hungerford, FRCS, Department of Ophthalmology, St Bartholomew's Hospital, West Smithfield, London ECIA 7BE.

Accepted for publication 17 August 1992
EBI rarely disappear completely and most leave a residual mass. The ophthalmoscopic appearance of successfully treated tumours has been classified into three regression patterns. ${ }^{6}$ In Type

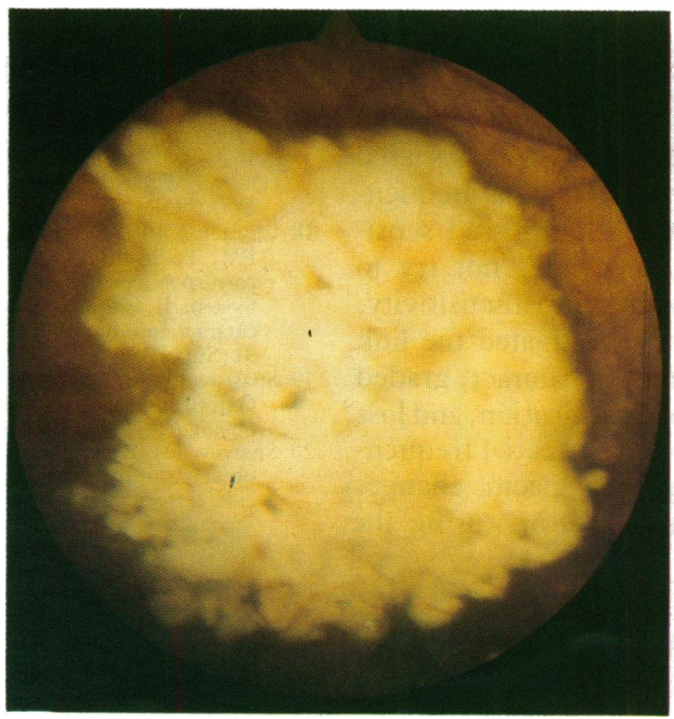

Figure 1 Fundus appearance of Type I regression pattern.

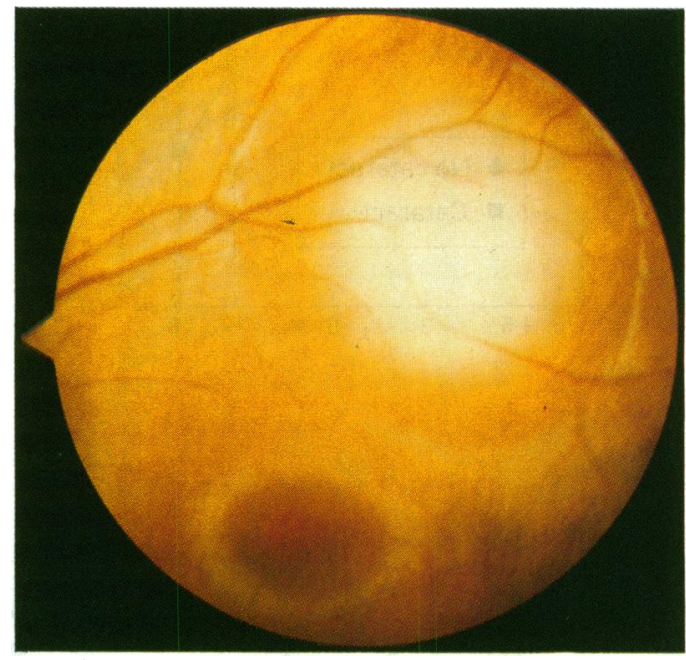

Figure 2 Fundus appearance of Type II regression pattern.

I regression the tumour assumes a glistening white appearance resembling cottage cheese (Fig 1). In Type II the regressed tumour has a homogeneous, grey, translucent aspect which has been likened to fish flesh and which is difficult to distinguish from viable tumour (Fig 2). Type III regression is a combination of Types I and II (Fig 3). Occasionally, EBI is followed by complete disappearance of tumours less than $3 \mathrm{~mm}$ in diameter ${ }^{78}$ with minor changes in the retinal pigment epithelium as the only indication of the original tumour site. This pattern has been termed Type 0 . Similar regression patterns have been described following radioactive scleral plaque therapy for retinoblastoma. ${ }^{9}$ After plaque therapy, total tumour destruction may result in the formation of a flat white scar when complete

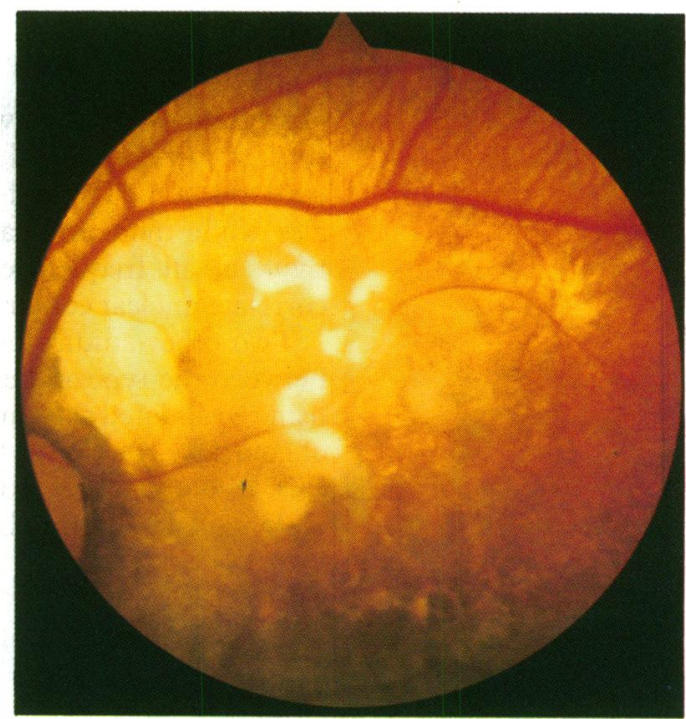

Figure 3 Fundus appearance of Type III regression pattern. 
retinal and choroidal atrophy reveals the underlying sclera. This pattern of regression is not seen after EBI and has been termed Type IV.'

The factors that influence regression pattern are not known. This study was undertaken to evaluate possible factors and to establish whether there is a relationship between regression pattern following EBI for retinoblastoma and tumour recurrence after treatment.

\section{Patients and methods}

A retrospective review was conducted of the records of all children who were treated for retinoblastoma by EBI at St Bartholomew's Hospital between January 1970 and December 1989. Only those tumours which fulfilled the following criteria were included in the study: (1) EBI was the primary treatment of retinoblastoma; (2) the tumour regressed following treatment; (3) there was a minimum follow up of 1 year. Many eyes had contained more than one retinoblastoma and the regression pattern and local tumour control status were determined for each individual lesion. One hundred and eighty tumours in 104 eyes of 83 children form the basis of this study.

All radiotherapy was carried out using megavoltage photon apparatus. Before 1985, EBI was always administered by a whole eye technique using either a telecobalt machine to deliver an anterior field when only one eye required treatment or a linear accelerator to deliver parallel, opposed lateral fields when both eyes were to be treated. A lateral linear accelerator field was also used when the first eye had been removed. ${ }^{10}$ Since 1985, a lens-sparing technique has been employed in selected cases. ${ }^{11}$ The dose prescribed ranged between 3500 cGy ( 35 Gy) in nine fractions over 3 weeks and $5000 \mathrm{cGy}$ in 22 fractions over $4 \frac{1}{2} 2$ weeks. The horizontal diameter of each retinoblastoma was estimated ophthalmoscopically before treatment and the tumour was assigned to one of three groups: small (diameter less than $6 \mathrm{~mm}$ ), medium-sized (diameter 6-12 mm), and large (diameter more than $12 \mathrm{~mm}$ ). After radiotherapy, each regressed tumour was ascribed to one of the four patterns 0 to III as outlined above.

Statistical analysis of the data was performed to answer the following questions: (1) what factors if any predict the regression pattern; and (2) does the pattern of regression have any bearing on the incidence of local tumour recurrence? The data were analysed using both a univariate ( $\chi^{2}$ test) and a multivariate (discriminant analysis) approach and tables were constructed for each possible predictor against regression pattern. The variables analysed were patient age and sex, the presence or absence of a family history of retinoblastoma, tumour size, method of EBI (whole-eye or lens-sparing), radiotherapy portal (anterior or lateral), the radiation dose prescribed, and the number of fractions employed.

Factors predicting recurrence were analysed by means of log rank analysis ${ }^{12}$ and shown as Kaplan-Meier plots. Cox model regression analysis ${ }^{13}$ was performed to look for independent predictors of recurrence. Relative risks were

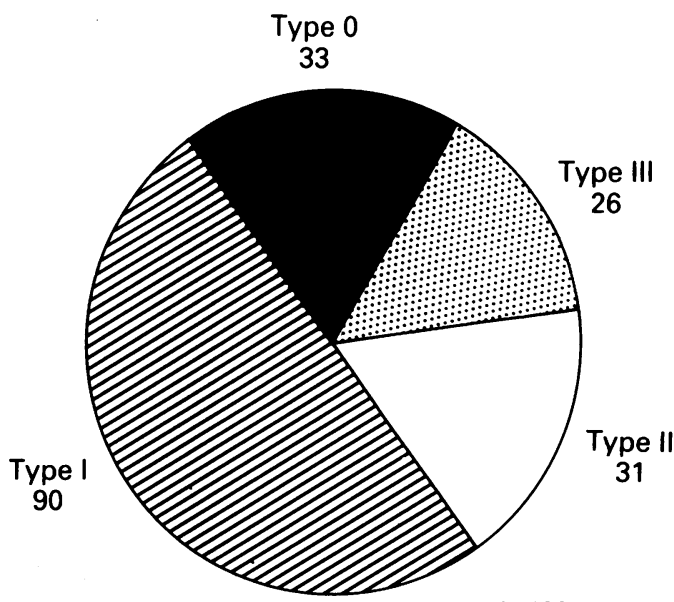

Figure 4 Distribution of regression patterns in 180 tumours.

calculated using the coefficient from the Cox analysis. Since initial tumour size appeared to determine subsequent outcome, other factors associated with size at diagnosis including the age of the patient were also analysed using the $\chi^{2}$ test.

\section{Results}

Forty three (52\%) of the children with retinoblastoma were male and $40(48 \%)$ were female; nine (11\%) had unilateral retinoblastoma and $74(89 \%)$ had bilateral disease. There was a positive family history of retinoblastoma in 31 cases (37\%). The average age at diagnosis was 15 months for unilateral tumours treated with radiotherapy (range 0.3-27 months) and 8 months for bilateral tumours (range $0 \cdot 1-50$ months). The median duration of follow up was 60 months (range 12-245 months). The distribution of regression patterns in the 180 tumours is shown in Figure 4. Type I regression was the commonest with 90 tumours (50\%) assigned to this group. Type 0, II, and III were approximately equally distributed with respectively 33 , 31 , and 26 tumours. In 21 tumours $(25 \%)$ with Type II (eight) and Type III regression (13) the pattern changed with time (Table 1). Type II regressed tumours converted mainly to Type 0 (six tumours) over a median interval of 22 months (range 5-33 months). Type III regressed tumours converted mainly to Type I (nine tumours), and Type 0 (three tumours). This conversion took place over a median interval of 27 months (range 6-83 months).

The distribution of regression patterns after EBI respectively for small, medium-sized, and large tumours is shown in Figure 5. A Type I regression pattern was seen following EBI of retinoblastoma lesions of all sizes but most large tumours (36 of 46 or $78 \%$ ) regressed in this pattern. Types II and III regression were seen

Table 1 Changes in regression pattern

\begin{tabular}{llllllll}
\hline & & \multicolumn{5}{c}{$\begin{array}{l}\text { Final pattern of those } \\
\text { changing }\end{array}$} \\
\cline { 4 - 7 } $\begin{array}{l}\text { Initial } \\
\text { pattern }\end{array}$ & $\begin{array}{l}\text { Total } \\
\text { no }\end{array}$ & $\begin{array}{l}\text { No } \\
\text { changing }\end{array}$ & $I$ & $I I$ & $I I I$ & 0 \\
\hline Type 0 & 31 & 0 & - & - & - & - \\
Type I & 90 & 0 & - & - & - & - \\
Type II & 33 & 8 & 1 & - & 1 & 6 \\
Type III & 26 & 13 & 9 & 1 & - & 3 \\
\hline
\end{tabular}


Figure 5 Distribution of regression patterns after external beam irradiation of small, medium-sized, and large retinoblastomas.

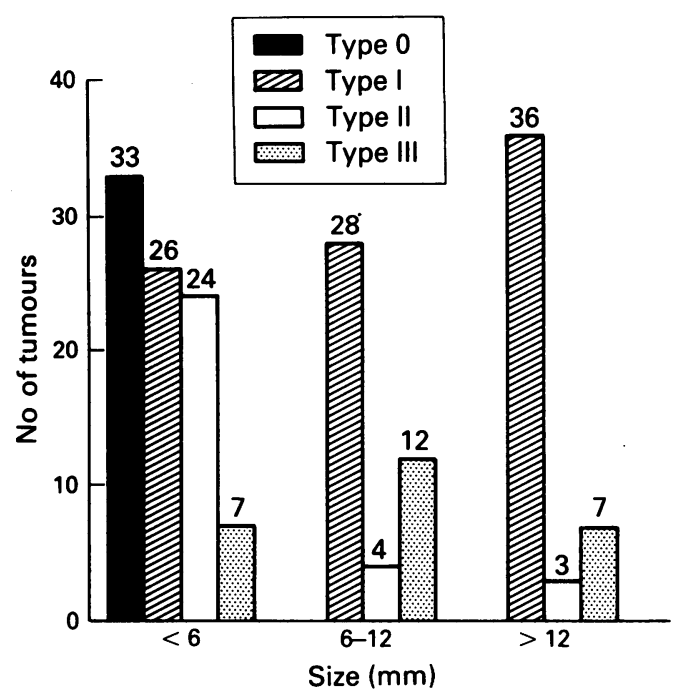

mainly in small and medium-sized retinoblastomas and Type 0 only after treatment of small tumours.

Univariate analysis of factors influencing regression pattern was performed and the results of $\chi^{2}$ tests are given in Table 2. By univariate analysis the pattern of regression was found to depend on the size of the tumour, on the method of treatment either by a whole eye or by a lens sparing approach, on the employment of an anterior or a lateral treatment portal, on the use of a total radiation dose of 350-390 Gy or one of 400-500 Gy, and on whether the chosen dose was given in $9-13$ or in $20-22$ fractions. When the data were examined in a multivariate fashion by discriminant analysis only tumour size, treatment method, and portal appeared as factors predicting regression pattern. The best coding of size was found to be one using a cut off at $6 \mathrm{~mm}$. From the discriminant model including tumour

Table 2 Univariate analysis of factors influencing regression pattern

\begin{tabular}{lccc}
\hline Variable & $\chi^{2}$ & $d f$ & $p$ \\
\hline Age & 9.3 & 6 & 0.2 \\
Family history & 2.7 & 3 & 0.4 \\
Sex & 0.8 & 3 & 0.9 \\
Laterality & 9.7 & 3 & 0.02 \\
Tumour size & 67.0 & 6 & $<0.001$ \\
Treatment approach & 37.0 & 3 & $<0.001$ \\
Treatment portal & 34.9 & 3 & 0.09 \\
Dose & 16.7 & 3 & 0.001 \\
Number of fractions & 23.2 & 3 & $<0.001$ \\
\hline
\end{tabular}

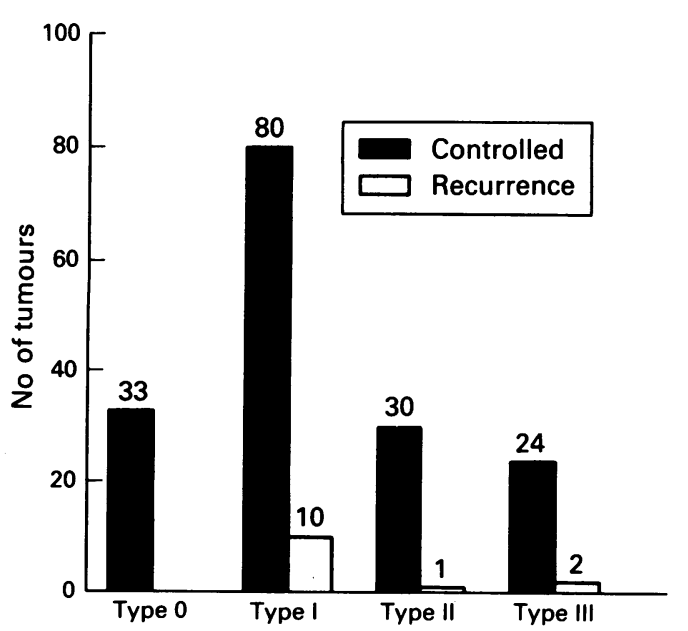

size, treatment method and portal, the accuracy of predicting each regression pattern was calculated. The classification value for Type 0 regression was the highest and the overall accuracy was $58 \%$.

Thirteen of the 180 tumours $(7 \%)$ recurred. The distribution of these recurrent tumours among the various regression patterns is shown in Figure 6. Type I regression had the largest number of recurrences (10 out of 13) whereas only one tumour recurred after Type II and two after Type III regression. The median interval between completion of EBI and recurrence was 12 months (range 5-40 months). Figure 7 shows a Kaplan-Meier plot of overall remission duration. Approximately $50 \%$ of recurrences were within 12 months and $90 \%$ within 32 months. No recurrences took place more than 40 months after completion of EBI. The distribution of recurrence by age at which the tumour recurred is shown in Figure 8. Approximately $50 \%$ of recurrent tumours had presented by age 27 months and $90 \%$ by 37 months. No recurrences took place after 48 months. Most (77\%) of the recurrences developed when the children were between 24 and 36 months of age.

A log rank analysis was performed to look for factors predicting recurrence. It showed tumour size and regression pattern to be predictive. Using Cox regression analysis, tumour size was the only independent predictor of recurrence (coefficient $=0.19, \quad \mathrm{SE}=0.056, \quad$ coeff $/ \mathrm{SE}=3.4$ equating this to normal deviate, $p=0.006$ ). Since there were few recurrences in this series, the power of the analysis is low. This means that some lesser predictive factors may have been missed. Using the coefficient from the Cox analysis, the relative risk for recurrence of a $12 \mathrm{~mm}$ tumour is $3 \cdot 2$ times that for a $6 \mathrm{~mm}$ lesion. The $95 \%$ confidence interval for this relative risk is $(2 \cdot 8,3 \cdot 5)$. Figure 9 shows KaplanMeier plots of the relapse-free interval following EBI for each tumour size category. Eight out of the 13 recurrences were following treatment of large tumours and the remaining five of mediumsized tumours. None of the small tumours recurred. These observations are statistically significant $(\mathrm{p}<0.01)$ using Cox model regression analysis. Tumour size is independently related to the age at diagnosis $(\mathrm{p}=0 \cdot 01)$.

\section{Discussion}

This series of 180 tumours is biased towards bilateral (89\%) and familial (37\%) cases. This is because nearly two thirds of all bilateral cases in the United Kingdom are initially treated at St Bartholomew's Hospital whereas only one third of unilateral cases are referred there. ' Furthermore, unilateral retinoblastoma is more often treated by enucleation than by EBI.

Since the original description and classification of regression patterns, the literature contains little information about the factors influencing the pattern of regression adopted by a particular retinoblastoma lesion or about the subsequent behaviour of the various types of regressed tumour. There are no published data on the distribution or incidence of recurrence following regression induced by EBI. Ellsworth concluded
Figure 6 Distribution of regression pattern. 


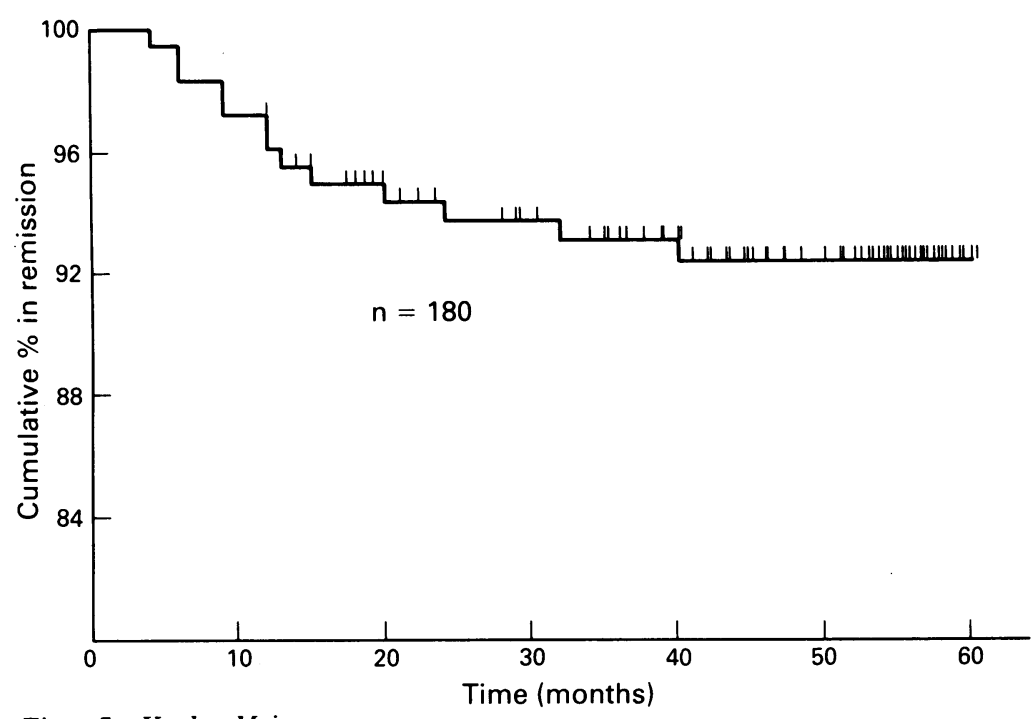

Figure 7 Kaplan-Meier plot of the regression duration.

Figure 9 Kaplan-Meier plot of the relapse-free interval following external beam irradiation for each tumour-size category: $s=\operatorname{small}(0-6 \mathrm{~mm})$, $m=$ medium-sized $(6 \cdot 1-12 \mathrm{~mm})$, and $l=$ large $(>12 \mathrm{~mm})$.

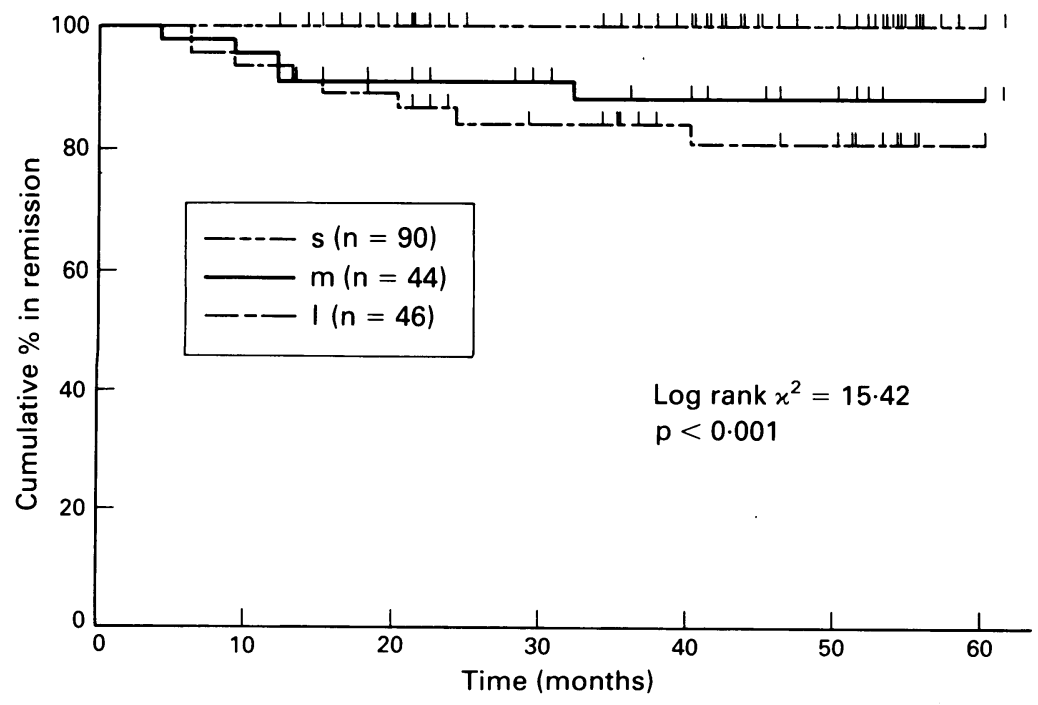

that Type I is the commonest regression pattern. ${ }^{7}$ Subsequently, Abramson suggested that Type III is commoner than Type $\mathrm{I} .{ }^{8}$ In our series $50 \%$ of tumours underwent Type I regression. There are two possible explanations for these differing incidences. The first is the significant $(p<0.01)$ finding of this study that initial tumour size is the only independent predictor of regression pattern. The use of focal treatments, and especially radioactive scleral plaques, to treat smaller tumours is likely to have led to a higher proportion of large retinoblastoma lesions treated by EBI in the St Bartholomew's Hospital series. Secondly, the differences may be due to the change observed in this study from one pattern to another, especially in Types II and III. The underlying basis of this change is the subject of a further study but, in essence, it is due to gradual loss of the Type II residual tissue. Thus, pure Type II regressed tumours changed to become Type 0 regressed lesions and Type III tumours lost the Type II element to become reclassified as Type I (Table 1).

On univariate analysis, tumour size appears to be the main factor determining regression pattern. Multivariate analysis showed that both the type of radiotherapy (whole eye or lens sparing) and the portal used (anterior or lateral) are also predictive of regression pattern. Possible

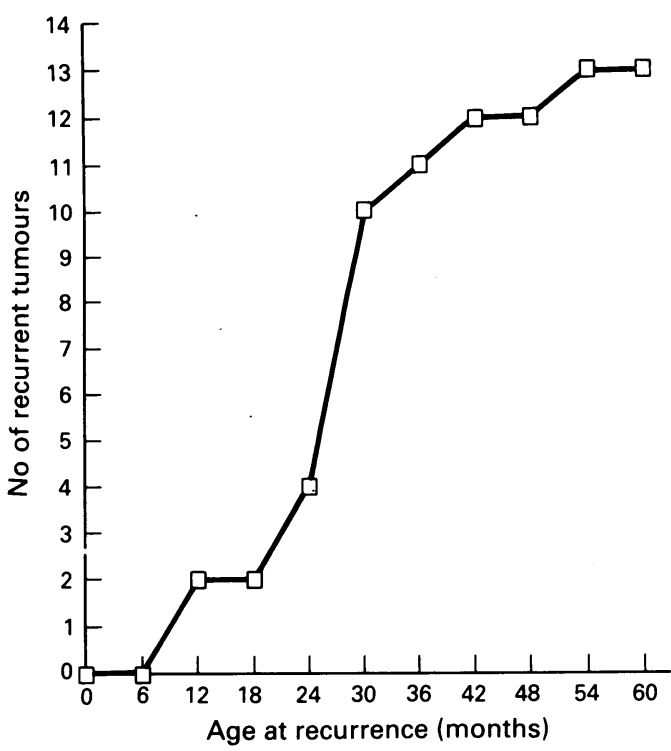

Figure 8 Tumour recurrence correlated with chronological age.

explanations for this finding may be that these two factors are significantly dependent on tumour size and location and that there has recently been a trend away from whole eye radiotherapy for smaller, posterior tumours.

According to Abramson, ${ }^{8}$ Type III regressed retinoblastomas have a tendency to recurrence whilst Type I regressed lesions rarely reactivate. In our study, 13 tumours recurred (7\%). Although 10 of the recurrences were in Type I regressed retinoblastomas, Cox regression analysis showed tumour size to be the only independent predictor of recurrence. Since the number of recurrences is small, it is possible that some lesser predictors may have been missed. However, from this analysis, there appears to be no direct relationship between regression pattern and recurrence. Both regression pattern and the risk of recurrence are dependent on initial tumour size and each relationship is statistically significant $(p<0.01)$. This means that large tumours tend to undergo Type I regression and also have a tendency towards recurrence. Ts'o et $a l^{14}$ suggested that the response of a retinoblastoma to EBI reflected the degree of differentiation of the tumour cells. Clinicohistological correlation in four of their tumours which exhibited 'fish flesh' regression pattern demonstrated marked photoreceptor differentiation with gliosis. These tumours did not recur and were considered to be relatively benign. In a separate group of four cases which recurred, but for which the pattern of regression is not stated, undifferentiated cells were seen within areas of photoreceptor differentiation suggesting a possible mechanism for recurrence. It has been suggested that tumours which undergo Type I regression are composed of less differentiated cells because of their more dramatic response to EBI. ${ }^{78}$ The expected higher risk of recurrence of Type I compared with Type II regressed tumours was not seen in our study. Instead, the relative risk of recurrence was solely dependent on the initial tumour size. This might be explained by larger tumours being composed of cells which are more malignant and which therefore have a greater capacity to proliferate and attain a large volume. In retinoblastoma, 
tumour size has long been recognised to be prognostic for ocular survival and, together with location anterior or posterior to the equator, forms the basis of the Reese-Ellsworth classification. ${ }^{15}$

There seems to be a spectrum of responses which a retinoblastoma may show, ranging from failure to regress through regression Types I to III with residual inactive tissue to complete disappearance of the tumour in regression Type 0 . It appears that this progression is dependent on tumour size.

All recurrences took place within 40 months of completion of EBI and none recurred after age 48 months. The observation that $77 \%$ of recurrences developed in children aged between 24 and 36 months is relevant when planning the intervals between follow up examinations and we have recently increased the frequency of such examinations from twice to three times during the third year.

Presented in part at the Fifth International Symposium on Retinoblastoma, 25-27 October 1990, Pine Mountain, GA, USA.

1 Sanders BM, Draper GJ, Kingston JE. Retinoblastoma in Great Britain 1969-80: incidence, treatment, and survival. Brf Ophthalmol 1988; 72: 576-83.
2 Shields JA, Shields CL, Donoso LA, Lieb WA. Changing concepts in the management of retinoblastoma. Ophthalmic Surg 1990; 21: 72-6.

3 Stallard $\mathrm{HB}$. The conservative treatment of retinoblastoma. Trans Ophthalmol Soc UK 1962; 82: 473-535.

4 Shields JA Augsburger JJ. Current approaches to the diagnosis and management of retinoblastoma. Surv Ophthalmol 1981; 25: 347-72.

5 Hilgartner HL. Report of a case of double glioma treated by Xrays. Tex Med f 1903; 18: 322

6 Dunphy EB. The story of retinoblastoma. Am 7 Ophthalmo 1964; 58: 539-52.

7 Ellsworth RM. The practical management of retinoblastoma. Trans Am Ophthalmol Soc 1969; 67: 462-534.

8 Abramson DH, Jereb B. Ellsworth RM. External beam radiation for retinoblastoma. Bull N Y Acad Med 1981; 57: 787-803.

9 Buys RJ, Abramson DH, Ellsworth RM, Haik B. Radiation regression patterns after cobalt plaque insertion for retinoregression patterns after cobalt plaque insertion
blastoma. Arch Ophthalmol 1983; 101: 1206-8.

10 Hungerford J, Kingston J, Plowman N. Tumours of the eye and orbit. In: Voûte PA, Barrett A, Bloom HJG, Lemerle J, Neidhardt MK, eds. Cancer in children. Berlin: Springe Verlag, 1986: 223-37.

11 Harnett AN, Hungerford JL, Lambert GD, Hirst A, Darlison $\mathrm{BL}$, Hart B, et al. Improved external beam radiotherapy fo the treatment of retinoblastoma. Brf Radiol 1987; 60: 75360

12 Peto R, Pike MC, Armitage P, Breslow NE, Cox DR, Howard JV, et al. Design and analysis of randomised clinical trials requiring prolonged observation of each patient. II. Analysis requiring prolonged observation of each pa.

13 Cox DR. Regression models and life tables. $7 R$ Stat Soc $B$ 1972; 34: 187-202.

14 Ts'o MOM, Zimmerman LE, Fine BS, Ellsworth RM. A cause of radioresistance in retinoblastoma: photorecepto differentiation. Trans Am Acad Ophthalmol Otolaryngol 1970; 74: 959-69.

15 Reese AB, Ellsworth RM. Evaluation and current concept of retinoblastoma therapy. Trans Am Acad Ophthalmo Otolaryngol 1963; 67: 164-72. 